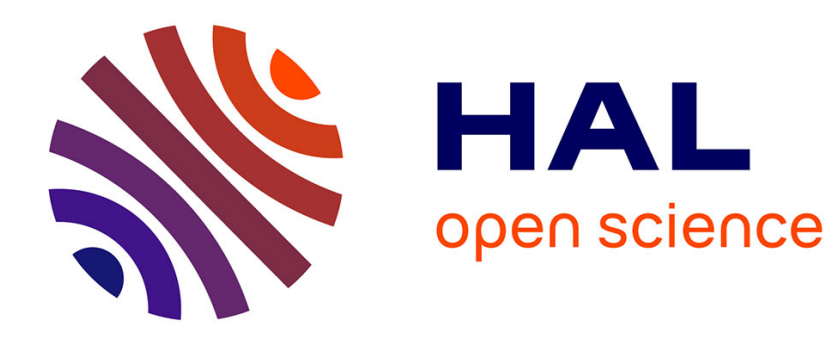

\title{
On Vertex Partitions of Hypercubes by Isometric Trees
}

Michel Mollard

\section{To cite this version:}

Michel Mollard. On Vertex Partitions of Hypercubes by Isometric Trees. SIAM Journal on Discrete Mathematics, 2011, 25, pp.534-538. hal-00535683v2

\section{HAL Id: hal-00535683 \\ https://hal.science/hal-00535683v2}

Submitted on 14 Mar 2011

HAL is a multi-disciplinary open access archive for the deposit and dissemination of scientific research documents, whether they are published or not. The documents may come from teaching and research institutions in France or abroad, or from public or private research centers.
L'archive ouverte pluridisciplinaire HAL, est destinée au dépôt et à la diffusion de documents scientifiques de niveau recherche, publiés ou non, émanant des établissements d'enseignement et de recherche français ou étrangers, des laboratoires publics ou privés. 


\title{
On Vertex Partitions of Hypercubes by Isometric Trees
}

\author{
Michel Mollard* \\ Institut Fourier \\ 100, rue des Maths \\ 38402 St Martin d'Hères Cedex FRANCE \\ michel.mollard@ujf-grenoble.fr
}

February 28, 2011

\begin{abstract}
When $n=2^{m}-1$ M.Ramras proved, by a counting argument, that for any isometrically embedded tree $T$ on $n$ edges in $Q_{n}$ there exists a group of translations $G$ such that $\{g(T) ; g \in G\}$ is a vertex partition of $Q_{n}$. Considering a more general context we are able to give an explicit construction of $G$ and can construct non group vertex partitions by isometric trees. We extend also this problem to vertex partition of $Q_{n^{\prime}}$ by translates of an isometrically embedded tree on $n=2^{m}-1$ edges for any $n^{\prime} \geq n$.
\end{abstract}

Keywords: Graph, Perfect code, Hypercube, Vertex Partition, Tiling.

\section{Introduction}

Twenty years ago M.Ramras [5] published a paper where he answered the following question of D. Rogers: If $n=2^{m}-1$ does the hypercube $Q_{n}$ have a vertex partition into antipodal paths? M.Ramras gave an explicit construction of such a partition. For this purpose he exhibited a set of generators of a subgroup $G$ of the group of translations $\Sigma\left(Q_{n}\right) \subset \operatorname{Aut}\left(Q_{n}\right)$, such that the set of translates $\{g(P) ; g \in G\}$ of the vertex set of $P$ of an antipodal path is the desired vertex partition.

He extended this result, proving, via a counting argument, that for any tree on $n=2^{m}-1$ edges isometrically embedded in $Q_{n}$ with vertex set $P$ there exists a subgroup $G$ of $\Sigma\left(Q_{n}\right)$ such that the set of translates $\{g(P) ; g \in G\}$ is a vertex partition. Notice that, in the general case, the author's method does not give an explicit construction of $G$.

This nice result generalizes the existence of perfect single-error-correcting codes constructed first by R.W.Hamming [3]. In this case, we take as tree the star $K_{1, n}$.

*CNRS and Université Joseph Fourier 
Notice that J.L.Vasiliev [6] constructed, for $n=2^{m}-1, n \geq 15$, perfect codes which are not equivalent to linear codes, i.e. vertex partitions by stars such that the set of translations $G$ is not a subgroup, or the translate of a subgroup, of the group of translations $\Sigma\left(Q_{n}\right)$.

In another series of papers M.Ramras considers edges partitions of $Q_{n}$ into isomorphic trees. More recent work have been done of this subject but is seems that this is not the case for vertex partitions. Both problems arise in the context of parallel computing and thus it will be interesting to improve our knowledge of vertex partitions.

Our goal at the beginning of this work was to prove the existence of non group partitions into antipodal paths. It seems also interesting, for the general case of trees, to give an explicit construction of a group, or more generally a set, of translations $G$. It would be also nice, for the case of the path $P_{n}$ to understand how the group proposed by M.Ramras can be derived from the Hamming code. In fact we found that all these problems can be easily solved, using elementary linear algebra. We arrive at the conclusion that looking for a vertex partition of $Q_{n}$ by translates of an isometrically embedded tree on $n$ edges is a problem independent, in some sense, of the choice of the tree, thus is equivalent to looking for a perfect code.

\section{Definitions and main result}

Let $\mathbb{F}^{n}$ be the vector space of dimension $n$ over the finite field $\mathbb{Z}_{2}$. The hypercube of dimension $n$ is the graph $Q_{n}$ whose vertices are the vectors of $\mathbb{F}^{n}$, and where two vertices are adjacent if they differ in exactly one coordinate.

The Hamming distance between two vectors $x, y \in \mathbb{F}^{n}, d(x, y)$ is the number of coordinates in which they differ. Notice that Hamming distance is the usual graph distance on $Q_{n}$.

The support of a vector $x$ is the set $\left\{i \in\{1,2, \ldots n\} ; x_{i} \neq 0\right\}$. The parity function is the function from $\mathbb{F}^{n}$ to $\mathbb{Z}_{2}$ defined by $\pi\left(x_{1}, x_{2}, \ldots, x_{n}\right)=x_{1}+x_{2}+\ldots+x_{n}$.

A perfect code, or more precisely a perfect single-error-correcting code of length $n$ is a set $C$ of vertices of $Q_{n}$ such that every vertex $x \in V\left(Q_{n}\right)$ is at distance at most 1 of exactly one element of $C$.

Two codes $C$ and $C^{\prime}$ are called equivalent if $C^{\prime}$ can be obtained from $C$ by an automorphism of $Q_{n}$, thus by applying a translation from a fixed vector and a fixed permutation of the coordinates. Using a translation of all the perfect code vectors by one of them we can always assume that the zero vector $\boldsymbol{O}$ belongs to the code.

Let $e_{1}, e_{2}, \ldots, e_{n}$ be the standard basis of $\mathbb{F}^{n}$, thus $e_{i}$ denotes the vector with just one single non zero coordinate position $i$. Let $e_{0}=\mathbf{0}$ be the zero vector and let $\mathbf{1}=(11 \ldots 1)$. Denote by $B_{n}$ the set $\left\{e_{0}, e_{1}, e_{2}, \ldots, e_{n}\right\}$. The direction of an edge $x y$ of $Q_{n}$ is the integer $i \in\{1,2, \ldots, n$,$\} such that y=x+e_{i}$.

For a subset $A$ of vectors of $\mathbb{F}^{n}$ and a vertex $x$, let $x+A$ be the set $\{x+a ; a \in A\}$. By definition of a perfect code $C=\left\{c_{1}, c_{2}, \ldots, c_{k}\right\}$ is a perfect code if and only if the sets $c_{1}+B_{n}, c_{2}+B_{n}, \ldots, c_{k}+B_{n}$ define a partition of $\mathbb{F}^{n}$ i.e.

- $\mathbb{F}^{n}=c_{1}+B_{n} \cup c_{2}+B_{n} \cup \ldots \cup c_{k}+B_{n}$

- $\forall i, j \in\{1,2, \ldots, k\} c_{i}+B_{n} \cap c_{j}+B_{n}=\emptyset$ 
This immediately leads to a necessary condition for the existence of $C$, the so-called packing condition, $2^{n}=|C|(n+1)$ thus $n=2^{m}-1$ for some $m$.

By analogy, G.Cohen, S.Litsyn, A.Vardy and G.Zémor [2] define a set $S$ as a tile of $\mathbb{F}^{n}$ if there exists a set $C=\left\{c_{1}, c_{2}, \ldots, c_{k}\right\}$ such that the sets $c_{1}+S, c_{2}+S, \ldots, c_{k}+S$ define a partition of $\mathbb{F}^{n}$. Notice that the definition is symmetric, $C$ is also a tile of $\mathbb{F}^{n}$, and they call the pair $(C, S)$ a tiling of $\mathbb{F}^{n}$.

R.W.Hamming [3] constructed, for any integer $m$, a linear subspace of $\mathbb{F}^{n}$, where $n=2^{m}-1$, which is a perfect code. It is easy to prove that all linear perfect codes are Hamming codes. In 1961 J.L.Vasiliev [6], and later many authors ([1, 4] for a survey) constructed perfect codes which are not linear codes.

Let $W=\left(v_{1}, v_{2}, \ldots, v_{n}\right)$ be a basis of $\mathbb{F}^{n}$. We will denote by $\theta_{W}$ be the automorphism of $\mathbb{F}^{n}$ defined by $\theta_{W}\left(\sum_{i=1}^{n} \lambda_{i} e_{i}\right)=\left(\sum_{i=1}^{n} \lambda_{i} v_{i}\right)$.

Lemma 1 Let $V=\left\{v_{0}, v_{1}, \ldots, v_{n}\right\}$ where $W=\left(v_{1}, v_{2}, \ldots, v_{n}\right)$ is a a basis of $\mathbb{F}^{n}$ and $v_{0}=\boldsymbol{O}$. Then $C=\left\{c_{1}, c_{2}, \ldots, c_{k}\right\}$ is a perfect code if and only if $\theta_{W}\left(c_{1}\right)+$ $V, \theta_{W}\left(c_{2}\right)+V, \ldots, \theta_{W}\left(c_{k}\right)+V$ is a partition of $\mathbb{F}^{n}$.

Proof : Notice that for any $j \in\{0,1, \ldots, n\}$ we have $\theta_{W}\left(e_{j}\right)=v_{j}$. By linearity for any $i, j$ we have $\theta_{W}\left(c_{i}\right)+v_{j}=\theta_{W}\left(c_{i}\right)+\theta_{W}\left(e_{j}\right)=\theta_{W}\left(c_{i}+e_{j}\right)$. Therefore, because $\theta_{W}$ is an automorphism, $\theta_{W}\left(c_{i}\right)+v_{j}=\theta_{W}\left(c_{i^{\prime}}\right)+v_{j^{\prime}}$ if and only if $c_{i}+e_{j}=c_{i^{\prime}}+e_{j^{\prime}}$. Assume that $C$ is a perfect code then the $\theta_{W}\left(c_{i}\right)+V$ are disjoint. Furthermore for any $x$ of $\mathbb{F}^{n}$ we knows that there exist $i \in\{1,2, \ldots, k\}$ and $j \in\{0,1, \ldots, n\}$ such that $\theta_{W}^{-1}(x)=c_{i}+e_{j}$, thus $x=\theta_{W}\left(c_{i}\right)+v_{j}$ and we have a partition.

Conversely if $\theta_{W}\left(c_{1}\right)+V, \theta_{W}\left(c_{2}\right)+V, \ldots, \theta_{W}\left(c_{k}\right)+V$ is a partition of $\mathbb{F}^{n}$ then the $\theta_{W}\left(c_{i}\right)+B_{n}$ are disjoint. Moreover for any $x$ of $\mathbb{F}^{n}$ there exist $i$ and $j$ such that $\theta_{W}(x)=\theta_{W}\left(c_{i}\right)+v_{j}$ and thus $x=c_{i}+e_{j}$.

For two graphs $G$ and $H$ an isometric embedding of $G$ in $H$ is a map $\alpha: V(G) \mapsto$ $V(H)$ which preserves distance. By extension we will denote by $\alpha(G)$ the subgraph of $H$ induced by $\alpha(V(G))$. If $G$ is injectively embedded in $Q_{n}$ we will say that there exists a vertex partition of $Q_{n}$ by $G$ if there exists a tiling of $V\left(Q_{n}\right)$ by $\alpha(V(G))$. It is immediate to check, as noticed by M.Ramras [5], that a tree $T$ is isometrically embedded in $Q_{n}$ if and only if no edges of $\alpha(T)$ use the same direction. If $\alpha$ is an isometric embedding in $Q_{n}$ then for any translation $t$ the map $\alpha^{\prime}=\alpha+t$ is also an isometric embedding. Therefore, if a graph $G$ is isometrically embeddable in $Q_{n}$ then, for any vertex $x$ of $G$ there exists an isometric embedding such that $\alpha(x)=\mathbf{0}$.

Lemma 2 Let $T$ be any tree on $p \leq n$ edges, and let $\alpha$ be an isometric embedding of $T$ in $Q_{n}$. Assume $\alpha(T)=\left\{\boldsymbol{O}, v_{1}, v_{2}, \ldots, v_{p}\right\}$ then the vectors $v_{1}, v_{2}, \ldots, v_{p}$ are linearly independent.

Proof : The proof is by induction on $p$. The result is clearly true when $p=1$ and assume it holds for any tree on $p-1$ edges. Let $x$ be a terminal vertex of $T$ and let $x y$ be the edge of $T$ incident to $x$. Consider the tree $T^{\prime}$ obtained by deletion of $x$ from $T$. We can always assume that $\alpha(x) \neq \mathbf{0}$ thus $\alpha(x)=v_{i}$ for some $i \in\{1,2, \ldots, p\}$. Let $j \in\{1,2, \ldots, n\}$ such that $\alpha(x)=\alpha(y)+e_{j}$. The restriction to $T^{\prime}$ of $\alpha$ is an isometric embedding thus the vectors $\left\{v_{k} ; k \in\{1,2, \ldots, p\}, k \neq i\right\}$ are independent by induction hypothesis. Notice that $e_{j}$ does not not appear in the 
basis decomposition of the $\left\{v_{k} ; k \in\{1,2, \ldots, p\}, k \neq i\right\}$. But $\alpha(y)$ belongs to this set and because $v_{i}=\alpha(y)+e_{j}$ the vector $v_{i}$ is also linearly independent of them.

Theorem 3 Let $T$ be any tree on $n=2^{m}-1$ edges, and let $\alpha$ be an isometric embedding of $T$ in $Q_{n}$. Assume $\alpha(T)=\left\{\boldsymbol{O}, v_{1}, v_{2}, \ldots, v_{n}\right\}$. Then the vectors $v_{1}, v_{2}, \ldots, v_{n}$ form a basis $W$ of $\mathbb{F}^{n}$. Furthermore if $C=\left\{c_{1}, c_{2}, \ldots, c_{k}\right\}$ is a perfect code of $Q_{n}$ then $\left\{\theta_{W}\left(c_{1}\right), \theta_{W}\left(c_{2}\right), \ldots, \theta_{W}\left(c_{k}\right)\right\}$ defines a vertex partition of $Q_{n}$ by the embedded tree $T$. All vertex partitions of $Q_{n}$ by $\alpha(T)$ can be obtained by this way.

Proof : By lemma $2 W$ is a basis of $\mathbb{F}^{n}$ and the result follows by lemma 1 .

Conversely if $S=\left\{s_{1}, s_{2}, \ldots, s_{k}\right\}$ defines a vertex partition of $Q_{n}$ by $T$ then $\left\{\theta_{W}^{-1}\left(s_{1}\right), \theta_{W}^{-1}\left(s_{2}\right), \ldots, \theta_{W}^{-1}\left(s_{k}\right)\right\}$ is a perfect code and thus all vertex partition of $Q_{n}$ by $T$ arise in this way.

Notice that the set $D=\left\{\theta_{W}\left(c_{1}\right), \theta_{W}\left(c_{2}\right), \ldots, \theta_{W}\left(c_{k}\right)\right\}$ is a linear subspace if and only if $C$ is linear. Furthermore if $b_{1}, b_{2} \ldots, b_{p}$ is a basis of $C$ then $\theta_{W}\left(b_{1}\right), \theta_{W}\left(b_{2}\right), \ldots, \theta_{W}\left(b_{p}\right)$ will be a basis of $D$.

Consider now a vertex partition of $Q_{n^{\prime}}$ by translates of an isometrically embedded tree on $n$ edges for some $n^{\prime} \geq n$. By the packing condition, $2^{n^{\prime}}=(n+1)|D|$, thus $n=2^{m}-1$ for some $m$.

Corollary 4 Let $T$ be any tree on $n=2^{m}-1$ edges, and let $\alpha$ be an isometric embedding of $T$ in $Q_{n^{\prime}}, n^{\prime} \geq n$. Then there exits a vertex partition of $Q_{n^{\prime}}$ by translates of the embedded tree $\alpha(T)$.

Proof : The vertices of $\alpha(T)=\left\{\mathbf{0}, v_{1}, v_{2}, \ldots, v_{n}\right\}$ define a subspace of dimension $n$. By a permutation of coordinates we can assume that this subspace is $V e c t\left(e_{1}, e_{2}, \ldots, e_{n}\right)$ thus there exist a vertex partition of $Q_{n}$ with set of translation say $D$. Then $D \cup\left\{e_{n+1}, e_{n+2} \ldots e_{n^{\prime}}\right\}$ define a vertex partition of $Q_{n^{\prime}}$ by translates of $\alpha(T)$.

\section{An example: antipodal paths}

The antipodal vertex of a vertex $x$ in $Q_{n}$ is the unique vertex $\bar{x}$ at distance $n$ of $x$. Notice that $\bar{x}=x+\mathbf{1}$. An antipodal path is a path in $Q_{n}$ of $n$ edges connecting some pair of antipodal vertices. We will say that an isometric embedding $\alpha(P)$ of an antipodal path in $Q_{n}$ is canonical if $v_{0}=\mathbf{0}$ and along the path the directions used are $1,2, \ldots, n$ in this order. We have thus, for any $i \in\{0,1, \ldots, n\}, v_{i}=\sum_{j=0}^{i} e_{j}$ and $\theta_{W}\left(\sum_{i=1}^{n} \lambda_{i} e_{i}\right)=\left(\sum_{i=1}^{n} \lambda_{i} \sum_{j=1}^{i} e_{j}\right)$. By a translation and a permutation of the coordinates we can always assume that an isometric embedding of $P$ is canonical.

A vector $u \in \mathbb{F}^{n}$ is of type 01 , respectively of type 10 , if there exists $i_{0} \in$ $\{0,1, \ldots, n\}$ such that $u=\sum_{i=i_{0}+1}^{n} e_{i}$, respectively $u=\sum_{i=1}^{i_{0}} e_{i}$. Notice that 1 and $\mathbf{0}$ are the only vectors of both types.

Lemma 5 If $u$ and $v$ are two distinct vectors both of type 10, or both of type 01 , then $u$ and $v$ differ by a set of consecutive coordinates. 
Proof : Assume first $u=\sum_{i=1}^{i_{0}} e_{i}$ and $v=\sum_{i=1}^{j_{0}} e_{i}$ for some $i_{0}, j_{0} \in\{0,1, \ldots, n\}$ Assume w.l.o.g. that $i_{0}<j_{0}$ we have thus $v=u+\sum_{i=i_{0}+1}^{j_{0}} e_{i}$. For the second case notice that if $u$ is a vector of type 01 then $u+\mathbf{1}$ is of type 10 .

Lemma 6 Let $\alpha(P)$ be an isometrically embedded antipodal path in $Q_{n}$ such that $\forall i \in\{0,1, \ldots, n\} v_{i}=\sum_{j=0}^{i} e_{j}$. Then a subset $C$ of $Q_{n}$ define a vertex partition by translates of $\alpha(P)$ if and only if

(i) $2^{n}=|C|(n+1)$ and

(ii) No pair of elements of $C$ differ by a set of consecutive coordinates.

Proof : Consider two translates of the embedded path say, $x+\alpha(P)$ and $y+\alpha(P)$. If $z$ is a common vertex of the two paths then for some $i, j \in\{0,1, \ldots, n\}$ we have $z=x+\sum_{k=1}^{i} e_{k}$ and $z=y+\sum_{k=1}^{j} e_{k}$. Thus by lemma 5 condition (ii) implies that the paths $x+\alpha(P)$ and $y+\alpha(P)$ are disjoint. By condition (i) every vertex belongs to some path.

If we assume that $C$ is a linear subspace, the last condition is equivalent to the condition used by Ramras:

(ii') No element of $C$ has as support a non-empty set of consecutive integers.

Let us recall Vasiliev's construction of perfect codes.

Theorem 7 (Vasiliev [6]) Let $C_{n}$ be a perfect code of length $n$. Assume $\boldsymbol{O} \in C_{n}$ and let $\lambda$ be a function from $C_{n}$ to $\mathbb{Z}_{2}$ such that $\lambda(\boldsymbol{O})=0$ and $\pi$ be the parity function. Then the set $C_{2 n+1}=\left\{(x, \pi(x)+\lambda(c), x+c) ; x \in \mathbb{F}^{n}, c \in C_{n}\right\}$ is a perfect code of length $2 n+1$.

Notice that if there exists $u, v \in C_{n}$ such that $\lambda(u+v) \neq \lambda(u)+\lambda(v)$ then $C_{2 n+1}$ is not equivalent to any linear code. Such a function $\lambda$ exists when $\left|C_{n}\right|>2$ thus there exists non linear codes when $n=2^{m}-1, n \geq 15$. If $\lambda(u)=0$ for any $u \in C$ we obtain the classical inductive construction of Hamming codes.

Theorem 8 Let $\alpha(P)$ be a canonical isometric embedding of an antipodal path in $Q_{n}$ and assume that $D_{n}$ defines a vertex partition by translates of $\alpha(P)$. Let $\gamma$ be a function from $D_{n}$ to $\mathbb{Z}_{2}$ such that $\gamma(\boldsymbol{O})=0$. Let $\Gamma$ be the function from $D_{n}$ to $\mathbb{F}^{n}$ defined by $\Gamma(d)=\boldsymbol{O}$ if $\gamma(d)=0$ and $\Gamma(d)=\mathbf{1}$ otherwise. Then the set $D_{2 n+1}=\left\{(y, \gamma(d), y+d+\Gamma(d)) ; y \in \mathbb{F}^{n}, d \in D_{n}\right\}$ defines a vertex partition of $\mathbb{F}^{2 n+1}$ by isometrically embedded antipodal paths.

Proof : Let us start by a direct proof using lemma 6. Notice first that $\left|D_{2 n+1}\right|(2 n+$ $2)=\left|D_{n}\right| 2^{n}(2 n+2)=\left|D_{n}\right|(n+1) 2^{n+1}=2^{2 n+1}$. Consider two vectors of $D_{2 n+1}$ say $u=(y, \gamma(d), y+d+\Gamma(d))$ and $u^{\prime}=\left(y^{\prime}, \gamma\left(d^{\prime}\right), y^{\prime}+d^{\prime}+\Gamma\left(d^{\prime}\right)\right)$. Assume that $u$ and $u^{\prime}$ differ by a set of consecutive coordinates.

- $\gamma(d)=\gamma\left(d^{\prime}\right)$. We have $y=y^{\prime}$ or $y+d=y^{\prime}+d^{\prime}$.

- If $y=y^{\prime}$ then $u+u^{\prime}=\left(\mathbf{0}, 0, d+d^{\prime}\right)$. But $d, d^{\prime} \in D_{n}$ thus by lemma 6 $d=d^{\prime}$ and $u=u^{\prime}$. 
- If $y+d=y^{\prime}+d^{\prime}$ then $u+u^{\prime}=\left(y+y^{\prime}, 0, \mathbf{0}\right)$. But $y+y^{\prime}=d+d^{\prime}$ and again by lemma $6 d=d^{\prime}, y=y^{\prime}$ and $u=u^{\prime}$.

- $\gamma(d) \neq \gamma\left(d^{\prime}\right)$ thus $u+u^{\prime}=\left(y+y^{\prime}, 1, d+y+d^{\prime}+y^{\prime}+\mathbf{1}\right)$. Therefore $y+y^{\prime}$ is of type 01 and $d+y+d^{\prime}+y^{\prime}+\mathbf{1}$ must be of type 10, thus $d+y+d^{\prime}+y^{\prime}$ of type 01. But $d+d^{\prime}=\left(y+y^{\prime}\right)+\left(d+y+d^{\prime}+y^{\prime}\right)$ then by lemma $5 d$ and $d^{\prime}$ must differ by a set of consecutive coordinates; a contradiction with lemma 6 .

We will now deduce theorem 8 from theorem 7 showing that this construction is in fact the analogue of Vasiliev's construction.

Alternative proof : Let $W=\left(v_{1}, v_{2}, \ldots, v_{n}\right)$ and $W^{\prime}=\left(v_{1}, v_{2}, \ldots, v_{2 n+1}\right)$, where $v_{i}=\sum_{j=1}^{i} e_{j}$. By theorem $3 D_{n}$ is obtained from a perfect code of length $n$ $C_{n}=\left\{\theta_{W}^{-1}(d) ; d \in D_{n}\right\}$. Let $\lambda: C_{n} \mapsto \mathbb{Z}_{2}$ defined by $\lambda(c)=\gamma\left(\theta_{W}(c)\right)$.

Consider $C_{2 n+1}=\left\{\left(\theta_{W}^{-1}(y), \pi\left(\theta_{W}^{-1}(y)\right)+\lambda\left(\theta_{W}^{-1}(d)\right), \theta_{W}^{-1}(y)+\theta_{W}^{-1}(d)\right) ; y \in \mathbb{F}^{n}, d \in D_{n}\right\}$. By theorem $7 C_{2 n+1}$ is a perfect code of length $2 n+1$ and thus by theorem $3 D_{2 n+1}$ defines a vertex partition of $\mathbb{F}^{2 n+1}$ by isometrically embedded antipodal paths where $D_{2 n+1}=\left\{\theta_{W^{\prime}}(c) ; c \in C_{2 n+1}\right\}$.

Notice that, for any $x \in \mathbb{F}^{n}$ and any $a \in \mathbb{Z}_{2}$ we have:

$$
\begin{aligned}
& \theta_{W^{\prime}}(x, 0, \mathbf{0})=\left(\theta_{W}(x), 0, \mathbf{0}\right)+\pi(x) \cdot(\mathbf{0}, 1, \mathbf{1}) \\
& \theta_{W^{\prime}}(\mathbf{0}, 0, x)=\left(\mathbf{0}, 0, \theta_{W}(x)\right) \\
& \theta_{W^{\prime}}(\mathbf{0}, a, \mathbf{0})=a .(\mathbf{0}, 1, \mathbf{1}) .
\end{aligned}
$$

Therefore:

$$
\begin{aligned}
& \theta_{W^{\prime}}\left(\theta_{W}^{-1}(y), 0, \mathbf{0}\right)=(y, 0, \mathbf{0})+\pi\left(\theta_{W}^{-1}(y)\right) \cdot(\mathbf{0}, 1, \mathbf{1}), \\
& \theta_{W^{\prime}}\left(\theta_{W}^{-1}\left(\mathbf{0}, \pi\left(\theta_{W}^{-1}(y)\right)+\lambda\left(\theta_{W}^{-1}(d)\right), \mathbf{0}\right)=\left[\pi\left(\theta_{W}^{-1}(y)\right)+\gamma(d)\right] \cdot(\mathbf{0}, 1, \mathbf{1})\right. \\
& \text { and } \theta_{W^{\prime}}\left(\mathbf{0}, 0, \theta_{W}^{-1}(y)+\theta_{W}^{-1}(d)\right)=(\mathbf{0}, 0, y+d) .
\end{aligned}
$$

By linearity of $\theta_{W^{\prime}}$ we obtain the expression of $D_{2 n+1}$.

Here also, if there exists $u, v \in D_{n}$ such that $\gamma(u+v) \neq \gamma(u)+\gamma(v)$ then $D_{2 n+1}$ is not a linear subspace. Notice that, if we set $\gamma(u)=0$ for any $u \in D_{n}$, we obtain a recursive construction of the linear subspace proposed by M.Ramras [5].

\section{Acknowledgment}

The author is grateful to M. Kovše for pointing him towards the work of M.Ramras.

\section{References}

[1] G. Cohen, I. Honkala, S. Litsyn and A. Lobstein, [1997]: Covering Codes, Chap. 11, Elsevier, Amsterdam.

[2] G. Cohen, S. Litsyn, A. Vardy and G. Zémor, [1996]: "Tilings of Binary spaces", SIAM J.Discrete Mathematics 9, pp 393-412.

[3] R. W. Hamming, [1950]: "Error detecting and error correcting codes", Bell Syst. Tech. J. 29, pp 147-160. 
[4] O. Heden, [2008]: "A survey on perfect codes", Advances in Mathematics of Communication 2(2), pp 223-247.

[5] M. Ramras, [1992]: "Symmetric Vertex Partitions of Hypercubes by Isometric Trees", Journal of Combinatorial Theory Series B 54, pp 239-248.

[6] J. L. Vasilev, [1962]: "On ungrouped, close-packed codes (in Russian)", Problemy Kibernet 8, pp 337-339. 